\section{Beryllium and Beryllium Oxide by XPS}

Christopher F. Mallinson ${ }^{\text {a) }}$ and James E. Castle

The Surface Analysis Laboratory, Department of Mechanical Engineering Sciences, University of Surrey, Guildford, GU2 7XH, UK

(Received 8 July 2013; accepted 1 October 2013; published 30 October 2013)

As received beryllium and beryllium oxide, purchased from Goodfellow, were analysed by x-ray photoelectron spectroscopy. Survey spectra, high resolution spectra of elemental peaks and beryllium Auger transitions were collected and are presented. The binding energy of metallic beryllium 1s peak and the beryllium oxide 1s peak were found to be 110.5 and $113.4 \mathrm{eV}$ respectively. The kinetic energy of the main metal and oxide KVV Auger transitions were found to be 103.5 and $95.5 \mathrm{eV}$ respectively. (C) 2013 American Vacuum Society.

[http://dx.doi.org/10.1116/11.20130701]

Keywords: beryllium Be; beryllium oxide BeO; x-ray photoelectron spectroscopy XPS

PACS: $82.80 . P v, 79.20 . F v, 61.50 . L t, 79.60 . B m$

\begin{tabular}{l}
\hline Accession \#s: 01265, 01266 \\
Technique: XPS \\
Host Material: \#01265: Be foil; \\
\#01266: BeO foil \\
Instrument: Thermo Scientific Theta \\
Probe \\
Major Elements in Spectra: Be, O, C \\
Minor Elements in Spectra: F, Ni, Ar, \\
Si, N, Zr, Ca, Cl, Na, Mg \\
Published Spectra: 9 \\
Spectra in Electronic Record: 38 \\
Spectral Category: comparison \\
\hline
\end{tabular}

\section{INTRODUCTION}

Beryllium is a metal with unusual properties that are exploited for certain very specific applications across a wide range of technologies. Examples are found in the use of copper beryllium alloys, where some $2 \%$ gives hardness and a spring function to copper, whilst retaining its good thermal and electrical conductivity (Ref. 1). These properties are exploited in hammers and other tools for mining applications, where the spark free behaviour is of particular value. At low levels in magnesium it can give resistance to high temperature oxidation, as was utilised in the Magnox alloy used to clad uranium in the first series of commercial nuclear power plants in the UK (Ref. 2). It has also found use in aluminium alloys where it has a hardening application. Its use in unalloyed form is most usually encountered in windows for x-ray detectors, e.g. in EDX energy analysers, a common tool in most materials laboratories. As a window it has excellent transmission combined with high strength as required for a vacuum component. Its high rigidity, low density and low coefficient of expansion have made it ideal for components in mirrors intended to operate in space (Ref. 3). The $\mathrm{Be}_{2}^{+}$ion is strongly polarizing because of its small radius and thus has a strong influence on the structure of minerals in which it is found. In the silicates, such as beryl, this action stabilises a cyclo-silicate ring structure, a highly strained structure that gives the silicon ion one of the lowest Auger parameter values found in the aluminosilicates (Ref. 4).

In spite of such a wide range of utilisation there are almost no published XPS spectra of $\mathrm{Be}$ or of $\mathrm{BeO}$ (Ref. 5). In this work we have attempted to provide these data using commercially available samples of both metal and the oxide. The metal is contaminated with low levels of fluorine, no doubt stemming from the extraction of Be from beryl via ammonium bifluoride and magnesium (Ref. 6). Other contaminants are also at a low level but are not thought to have had an impact on the Be spectrum. However, because of its high reactivity and use of high temperatures in its production as a solid metal, Be metal often carries an oxide film of a thickness, up to $10 \mathrm{~nm}$, that is very significant in XPS analysis (Ref. 7). Unfortunately the oxide, particularly in dust form, is highly toxic and thus the surface cannot be repolished under typical conditions found in a metallographic laboratory (Ref. 8). For

a) Author to whom correspondence should be addressed. this reason it is also desirable to minimise the time spent etching samples. In producing these data the surface has been etched using the in situ argon ion beam on the spectrometer. It is also noteworthy that, along with the oxide, a $\mathrm{C} 1 \mathrm{~s}$ component in the position expected for a carbonate is also found. This probably originates from air exposure during transport.

Finally, we have provided here the KVV Auger peaks of both $\mathrm{Be}$ and $\mathrm{BeO}$. Unfortunately they are of low kinetic energy and thus heavily influenced by the surface oxidation of the metal. A shift of $\sim 10 \mathrm{eV}$ is known to occur for the main KVV Auger peak upon oxidation of beryllium (Refs. 9 and 10). However, they are novel within the XPS literature and hence of potential value to those considering the origin of Auger spectra from low-atomic number elements. As a result of the presence of a carbonate it is clear that the $\mathrm{O} 1 \mathrm{~s}$ contains a significant contribution from this ligand. This is not resolvable as a component distinct from that of the $\mathrm{O} 1 \mathrm{~s}$ of $\mathrm{BeO}$. However, even when appropriate corrections are made for the presence of $\mathrm{BeCO}_{3}$ there is an excess of oxygen when using either the Scofield or the Wagner databases of relative sensitivity factors.

\section{SPECIMEN DESCRIPTION (ACCESSION \#01265, 1 OF 2) -}

Host Material: Be foil

CAS Registry \#: 7440-41-7

Host Material Characteristics: homogeneous; solid; polycrystalline; conductor; metal

Chemical Name: beryllium

Source: GoodFellow

Host Composition: Be

Form: foil, $1 \times 25 \times 25 \mathrm{~mm}, 99.0 \%$

Lot \#: BE000330

As Received Condition: Sample arrived vacuum packed wrapped in cardboard from GoodFellow. It was stored wrapped in aluminium foil under normal conditions.

Typical Analysis Provided by GoodFellow (ppm): $\mathrm{Al}<500$, B $<3, \mathrm{Cd}<2$, Ca $<100, \mathrm{C}<700, \mathrm{Cr}<100$, Co $<10, \mathrm{Cu}<100$, $\mathrm{Fe}<700, \mathrm{~Pb}<20, \mathrm{Li}<3, \mathrm{Mg}<500, \mathrm{Mn}<120$, Mo $<20$, Ni $<200, \mathrm{~N}<400, \mathrm{Si}<400, \mathrm{Ag}<10, \mathrm{BeO}<8000$.

Analyzed Region: not specified 
Ex Situ Preparation/Mounting: Sample was sonically cleaned in hexane for $10 \mathrm{~min}$ prior to analysis.

In Situ Preparation: Sample was Argon ion etched within the spectrometer for $60 \mathrm{~s}$ to remove carbonaceous contamination.

Charge Control: The beryllium binding energy values were corrected for $\mathrm{C} 1 \mathrm{~s}$ at $285.0 \mathrm{eV}$ using a shift of $-1.3 \mathrm{eV}$.

Temp. During Analysis: $300 \mathrm{~K}$

Pressure During Analysis: $<1.2 \times 10^{-7} \mathrm{~Pa}$

\section{SPECIMEN DESCRIPTION (ACCESSION \#01266, 2 OF 2) -}

Host Material: $\mathrm{BeO}$ foil

CAS Registry \#: 1304-56-9

Host Material Characteristics: homogeneous; solid; polycrystalline; dielectric; composite; ceramic

Chemical Name: beryllium oxide

Source: GoodFellow

Host Composition: $\mathrm{BeO}$

Form: foil, $1 \times 25 \times 25 \mathrm{~mm}, 99.5 \%$

Lot \#: $739-203-48$

As Received Condition: Sample arrived vaccum packed wrapped in cardboard from GoodFellow. It was stored wrapped in aluminium foil under normal conditions.

Analyzed Region: not specified

Ex Situ Preparation/Mounting: not specified

In Situ Preparation: Sample was Argon ion etched for $30 \mathrm{~s}$ although this appeared to have little effect on the level of surface carbon.

Pre-Analysis Beam Exposure: Total of $12 \mathrm{~h}$ previous exposure, $4 \mathrm{~h}$ within an ESCA Lab and 8 within a Theta Probe before these spectra acquired.

Charge Control: Flood gun used for charge neutralisation.

Electron energy $-3 \mathrm{~V}$

Extractor V $30 \mathrm{~V}$

Emission $100 \mathrm{uA}$

The beryllium oxide binding energy values were corrected for $\mathrm{C} 1 \mathrm{~s}$ at $285.0 \mathrm{eV}$ using a shift of $+1.1 \mathrm{eV}$.

Temp. During Analysis: $300 \mathrm{~K}$

Pressure During Analysis: $<1.2 \times 10^{-7} \mathrm{~Pa}$

\section{INSTRUMENT DESCRIPTION}

Manufacturer and Model: Thermo Scientific Theta Probe

Analyzer Type: spherical sector

Detector: multi-channel plate detector

Number of Detector Elements: 128

INSTRUMENT PARAMETERS COMMON TO ALL SPECTRA -

\section{Spectrometer}

Analyzer Mode: constant pass energy

Throughput $\left(T=E^{N}\right)$ : $N=$ See comment below
Throughput Comment: The transmission function is calculated from a cubic polynomial fit to a plot of LOG (Peak Area * $\mathrm{XSF} / \mathrm{PE})$ vs. LOG (RR) $\mathrm{RR}=\mathrm{KE} / \mathrm{PE}($ Retard Ratio $=$ Kinetic Energy/Pass Energy). XSF is a term to account for the difference in absolute sensitivities of the $\mathrm{Ag} \mathrm{MNN}$ and Ag 3d peaks.

Excitation Source Window: none

Excitation Source: $\mathrm{Al} K_{\alpha}$, monochromatic

Source Energy: $1486.6 \mathrm{eV}$

Source Strength: $200 \mathrm{~W}$

Source Beam Size: $200 \mu \mathrm{m} \times 200 \mu \mathrm{m}$

Signal Mode: pulse single channel

\section{Geometry}

Incident Angle: $30^{\circ}$

Source to Analyzer Angle: $67.4^{\circ}$

Emission Angle: $53^{\circ}$

Specimen Azimuthal Angle: $70^{\circ}$

Acceptance Angle from Analyzer Axis: $50^{\circ}$

Analyzer Angular Acceptance Width: $30^{\circ} \times 30^{\circ}$

\section{Ion Gun}

Manufacturer and Model: Thermo EX05

Energy: $3000 \mathrm{eV}$

Current: $2 \mathrm{~mA}$

Current Measurement Method: biased stage

Sputtering Species: $\mathrm{Ar}^{+}$

Spot Size (unrastered): $200 \mu \mathrm{m}$

Raster Size: $2000 \mu \mathrm{m} \times 2000 \mu \mathrm{m}$

Incident Angle: $45^{\circ}$

Polar Angle: $58.4^{\circ}$

Azimuthal Angle: $100^{\circ}$

\section{DATA ANALYSIS METHOD}

Energy Scale Correction: Plotted $\mathrm{Be}$ and $\mathrm{BeO}$ binding energies are corrected by setting the $\mathrm{C} 1 \mathrm{~s}$ peak to $285.0 \mathrm{eV}$.

Recommended Energy Scale Shift: The beryllium binding energy values (Accession \#1265) can be corrected using C 1s peak at $285.0 \mathrm{eV}$ using a shift of $-1.3 \mathrm{eV}$. The beryllium KVV spectra were collected separately and a shift of $-0.5 \mathrm{eV}$ is used for correction. The beryllium oxide binding energy values (Accession \#1266) can be corrected using C 1s peak at $285.0 \mathrm{eV}$ using a shift of $+1.1 \mathrm{eV}$.

Peak Shape and Background Method: The peak fitting function used is a Gaussian - Lorentzian product function with a maximum of $30 \%$ Lorentzian mix. The Shirley function was used for background substraction.

Quantitation Method: Quantification carried out using Avantage v4.84 software from Thermo Scientific. Sensitivity factors used in Thermo Scientific Avantage software. Peak library is ALWAG from C. D. Wagner, et al., Surf. Interface Anal., 3. 211 (1981). 


\section{ACKNOWLEDGMENTS}

The Authors wish to thank the EPRSC, the Engineering Doctorate Programme at the University of Surrey and the sponsorship of AWE.

\section{REFERENCES}

1. H. F. Silliman, Ind. Eng. Chem. 26(12), 1424-1428 (1936).

2. J. E. Castle, S. J. Gregg, J. E. Antill, and W. B. Jepson, J. Nucl. Mater. 5, 254-256 (1962).

3. R. R. Altenhof, Opt. Eng. 15, 153265-153274 (1976).
4. R. H. West and J. E. Castle, J. Electron Spectrosc. Relat. Phenom. 18(4), 355-358 (1980).

5. N. L. Kuehler, D. L. Williams, W. E. Moddeman, and J. C. Birkbeck, Surf. Interface Anal. 27(4), 273-282 (1999).

6. A. J. Stonehouse, J. Vac. Sci. Technol., A 4, 1163-1170 (1968).

7. A. J. Stonehouse and W. W. Beaver, Mater. Prot. 4, 24-28 (1965).

8. Toxicological Review of Beryllium and Compounds U.S. Environmental Protection Agency 1998.

9. V. Sashin, M. Bolorizadeh, A. Kheifets, and M. Ford, J. Phys.: Condens. Matter 15, 3567-3581 (2003).

10. E. Lejeune and R. Dixon, J. Appl. Phys. 43, 1998-1999 (1971). 
SPECTRAL FEATURES TABLE

\begin{tabular}{|c|c|c|c|c|c|c|c|}
\hline $\begin{array}{l}\text { Spectrum } \\
\text { ID \# }\end{array}$ & $\begin{array}{l}\text { Element/ } \\
\text { Transition }\end{array}$ & $\begin{array}{c}\text { Peak } \\
\text { Energy } \\
(\mathrm{eV})\end{array}$ & $\begin{array}{c}\text { Peak } \\
\text { Width } \\
\text { FWHM }(\mathrm{eV})\end{array}$ & $\begin{array}{l}\text { Peak Area } \\
(\mathrm{eV} \times \mathrm{cts} / \mathrm{s})\end{array}$ & $\begin{array}{l}\text { Sensitivity } \\
\text { Factor }\end{array}$ & $\begin{array}{l}\text { Concentration } \\
\text { (at. \%) }\end{array}$ & $\begin{array}{c}\text { Peak } \\
\text { Assignment }\end{array}$ \\
\hline $01265-02^{a}$ & $\mathrm{Be} 1 \mathrm{~s}$ & 110.5 & 1.17 & 1735 & 0.059 & 14.67 & Be metal \\
\hline $01265-02^{b}$ & $\mathrm{Be} 1 \mathrm{~s}$ & 113.5 & 1.88 & 3731 & 0.059 & 31.59 & $\mathrm{Be}$ in $\mathrm{BeO}$ \\
\hline $01265-02^{c}$ & Be 1s & 114.7 & 1.83 & 125 & 0.059 & 1.06 & $\mathrm{Be}$ in $\mathrm{BeCO}_{3}$ \\
\hline $01265-07^{d}$ & $\operatorname{Ar} 2 p$ & 241.9 & 2.2 & 2604 & 0.96 & 1.44 & $\mathrm{Ar}$ \\
\hline 01265-03 & C 1s & 285.0 & 1.68 & 4357 & 0.25 & 9.49 & Hydrocarbon surface contamination \\
\hline $01265-03^{e}$ & C 1s & 289.3 & 1.73 & 472 & 0.25 & 1.03 & $\mathrm{C}$ in $\mathrm{BeCO}_{3}$ \\
\hline $01265-03^{\dagger}$ & C 1s & 286.8 & 1.64 & 265 & 0.25 & 0.58 & Surface carbon contamination \\
\hline 01265-049 & O 1s & 531.4 & 2.01 & 9767 & 0.66 & 35.7 & $\mathrm{O}$ in $\mathrm{BeO}$ \\
\hline $01265-04^{h}$ & O 1s & 532.2 & 1.72 & 140 & 0.66 & 0.51 & Polar surface contamination \\
\hline $01265-04^{i}$ & O 1s & 533.0 & 1.88 & 801 & 0.66 & 2.93 & $\mathrm{O}$ in $\mathrm{BeCO}_{3}$ \\
\hline $01265-06^{\mathrm{a0}}$ & $\mathrm{F} 1 \mathrm{~s}$ & 686.2 & 2.92 & 1024 & 1 & 0.73 & $\mathrm{~F}$ in $\mathrm{BeF}_{2}$ \\
\hline $01265-08^{\text {aa }}$ & $\mathrm{Ni} 2 \mathrm{p}$ & 852.4 & 1.12 & 249 & 3 & 0.07 & Ni metal \\
\hline $01265-05^{\mathrm{ab}}$ & Be KVV & 103.5 & $\cdots$ & $\cdots$ & $\cdots$ & $\cdots$ & $\mathrm{Be} \mathrm{KVV}$ and $\mathrm{BeO} \mathrm{KVV}$ \\
\hline $01266-02^{\mathrm{ac}}$ & $\mathrm{Be} 1 \mathrm{~s}$ & 113.3 & 2.75 & 2461 & 0.059 & 21.67 & $\mathrm{Be}$ in $\mathrm{BeO}$ \\
\hline $01266-07^{\mathrm{ad}}$ & Si $2 p$ & 102.4 & 2.65 & 1529 & 0.27 & 2.93 & Silicone oil \\
\hline $01266-08^{a e}$ & $\operatorname{Zr} 3 d$ & 183.2 & 2.34 & 563 & 2.1 & 0.11 & $\mathrm{Zr}$ in $\mathrm{ZrO}_{2}$ \\
\hline $01266-09^{a f}$ & $\mathrm{Cl} 2 \mathrm{p}$ & 198.9 & 2.87 & 496 & 0.73 & 0.27 & Contamination \\
\hline 01266-05 & C 1s & 285.0 & 2.91 & 12999 & 0.25 & 29.5 & Hydrocarbon contamination \\
\hline $01266-10^{\text {ag }}$ & Ca $2 p$ & 351.0 & 3.09 & 1410 & 1.58 & 0.52 & $\mathrm{Ca}$ in $\mathrm{CaO}$ or possibly $\mathrm{CaCO}_{3}$ \\
\hline $01266-11^{\text {ah }}$ & $\mathrm{N} 1 \mathrm{~s}$ & 399.7 & 2.79 & 2431 & 0.42 & 3.50 & Contamination or impurity \\
\hline $01266-03^{\text {ai }}$ & O 1s & 531.4 & 2.86 & 19455 & 0.66 & 36.6 & $\mathrm{O}$ in $\mathrm{BeO}$ \\
\hline $01266-06^{\text {ah }}$ & $\mathrm{Na} 1 \mathrm{~s}$ & 1071.6 & 2.88 & 6808 & 2.3 & 3.52 & Contamination or impurity \\
\hline $01266-12^{\text {ag }}$ & $\operatorname{Mg} 1 \mathrm{~s}$ & 1303.7 & 3.21 & 2730 & 3.5 & 1.42 & $\mathrm{Mg}$ in $\mathrm{MgO}$ \\
\hline $01266-04^{\mathrm{bo}}$ & $\mathrm{Be}$ KVV & 95.5 & $\ldots$ & & $\cdots$ & $\cdots$ & $\mathrm{BeO}$ KVV \\
\hline
\end{tabular}

a Bulk beryllium metal

${ }^{\mathrm{b}}$ Oxide formed on the surface when exposed to atmosphere

${ }^{c}$ Beryllium in beryllium carbonate formed when the metal surface is exposed to atmosphere

${ }^{\mathrm{d}}$ Argon implanted as a result of etching the sample surface

${ }^{\text {e }}$ Carbon in beryllium carbonate formed when the metal surface is exposed to atmosphere

${ }^{f}$ Believed to be the contribution from polar surface contamination

g Oxygen in beryllium oxide formed when the metal surface is exposed to atmosphere

${ }^{\mathrm{h}}$ Oxygen believed to be associated with surface polar carbon contamination

'Oxygen in beryllium carbonate formed when the metal is exposed to atmosphere

${ }^{a} 0$ Impurity remaining from the manufacturing of beryllium metal

aa Impurity believed to be present at trace level from manufacturing beryllium metal

${ }^{\mathrm{ab}}$ Both oxide and metal components are present in the Auger transition region

${ }^{\text {ac }}$ Bulk beryllium oxide

${ }^{\text {ad }}$ Believed to be silicone oil contamination on the as-received sample surface

ae Zirconium present as small particles of zirconia embedded in the sample surface from polishing the sample surface during processing

af Believed to be surface contamination

ag Impurity believed to be from manufacturing

ah Possible impurity introduced during manufacturing

ai Excess oxygen concentration compared to beryllium concentration suggests a range of unresolvable oxygen containing components

${ }^{\text {bo }}$ Main Auger transition region for $\mathrm{BeO}$

\begin{tabular}{lccccccc}
\hline \multicolumn{7}{c}{ ANALYZER CALIBRATION TABLE } \\
\hline Spectrum & $\begin{array}{c}\text { Element/ } \\
\text { ID \# }\end{array}$ & $\begin{array}{c}\text { Peak } \\
\text { Energy } \\
(\mathbf{e V})\end{array}$ & $\begin{array}{c}\text { Peak } \\
\text { Width } \\
\text { FWHM (eV) }\end{array}$ & $\begin{array}{c}\text { Peak Area } \\
(\mathbf{e V} \times \mathbf{c t s} / \mathbf{s})\end{array}$ & $\begin{array}{c}\text { Sensitivity } \\
\text { Factor }\end{array}$ & $\begin{array}{c}\text { Concentration } \\
\text { (at. \%) }\end{array}$ & $\begin{array}{c}\text { Peak } \\
\text { Assignment }\end{array}$ \\
\hline 22 & $\mathrm{Au} 4 \mathrm{f}_{7 / 2}, 4 \mathrm{f}_{5 / 2}$ & 84.1 & 0.74 & 17347 & 4.95 & 100 & Au metal \\
31 & $\mathrm{Cu} 2 \mathrm{p}_{3 / 2}$ & 932.6 & 0.87 & 108427 & 4.2 & 100 & Cu metal \\
\hline
\end{tabular}




\begin{tabular}{|c|c|c|c|c|c|}
\hline \multicolumn{6}{|c|}{ GUIDE TO FIGURES } \\
\hline Spectrum (Accession) \# & Spectral Region & Voltage Shift & Multiplier & Baseline & Comment \# \\
\hline $1265-01$ & survey & 1.3 & 1 & 0 & 1 \\
\hline $1265-02$ & $\mathrm{Be} 1 \mathrm{~s}$ & 1.3 & 1 & 0 & 1 \\
\hline $1265-03$ & C 1s & 1.3 & 1 & 0 & 1 \\
\hline $1265-04$ & O 1s & 1.3 & 1 & 0 & 1 \\
\hline $1265-05$ & Be KVV & 0.5 & 1 & 0 & 1 \\
\hline $1265-06[N P]^{\star *}$ & F 1s & 1.3 & 1 & 0 & 1 \\
\hline $1265-07$ [NP] & $\operatorname{Ar} 2 p$ & 1.3 & 1 & 0 & 1 \\
\hline $1265-08$ [NP] & $\mathrm{Ni} 2 \mathrm{p}$ & 1.3 & 1 & 0 & 1 \\
\hline $1266-01$ & survey & -1.1 & 1 & 0 & 2 \\
\hline $1266-02$ & $\mathrm{Be} 1 \mathrm{~s}$ & -1.1 & 1 & 0 & 2 \\
\hline $1266-03$ & $01 \mathrm{~s}$ & -1.1 & 1 & 0 & 2 \\
\hline $1266-04$ & Be KVV & 0.5 & 1 & 0 & 2 \\
\hline $1266-05$ [NP] & C 1s & -1.1 & 1 & 0 & 2 \\
\hline 1266-06 [NP] & $\mathrm{Na} 1 \mathrm{~s}$ & -1.1 & 1 & 0 & 2 \\
\hline $1266-07$ [NP] & Si $2 p$ & -1.1 & 1 & 0 & 2 \\
\hline $1266-08$ [NP] & $\mathrm{Zr} 3 d$ & -1.1 & 1 & 0 & 2 \\
\hline 1266-09 [NP] & $\mathrm{Cl} 2 \mathrm{p}$ & -1.1 & 1 & 0 & 2 \\
\hline $1266-10$ [NP] & Ca $2 p$ & -1.1 & 1 & 0 & 2 \\
\hline $1266-11$ [NP] & $\mathrm{N} 1 \mathrm{~s}$ & -1.1 & 1 & 0 & 2 \\
\hline $1266-12$ [NP] & $\mathrm{Mg} 1 \mathrm{~s}$ & -1.1 & 1 & 0 & 2 \\
\hline $1267-01$ [NP] & survey & - & 1 & 0 & 3 \\
\hline 1267-02 [NP] & $\mathrm{Au} 4 \mathrm{f}$ & - & 1 & 0 & 3 \\
\hline $1267-03$ [NP] & $\mathrm{Au} 4 \mathrm{f}$ & - & 1 & 0 & 3 \\
\hline $1267-04$ [NP] & $\mathrm{Au} 4 \mathrm{f}$ & - & 1 & 0 & 3 \\
\hline $1267-05$ [NP] & $\mathrm{Au} 4 \mathrm{f}$ & - & 1 & 0 & 3 \\
\hline $1267-06$ [NP] & $\mathrm{Au} 4 \mathrm{f}$ & - & 1 & 0 & 3 \\
\hline 1267-07 [NP] & $\mathrm{Au} 4 \mathrm{f}$ & - & 1 & 0 & 3 \\
\hline $1267-08$ [NP] & $\mathrm{Au} 4 \mathrm{f}$ & - & 1 & 0 & 3 \\
\hline $1267-09$ [NP] & $\mathrm{Au} 4 \mathrm{f}$ & - & 1 & 0 & 3 \\
\hline $1268-01$ [NP] & survey & - & 1 & 0 & 4 \\
\hline $1268-02$ [NP] & Cu 2p & - & 1 & 0 & 4 \\
\hline $1268-03$ [NP] & Cu 2p & - & 1 & 0 & 4 \\
\hline 1268-04 [NP] & Cu 2p & - & 1 & 0 & 4 \\
\hline 1268-05 [NP] & Cu 2p & - & 1 & 0 & 4 \\
\hline $1268-06$ [NP] & Cu 2p & - & 1 & 0 & 4 \\
\hline 1268-07 [NP] & Cu $2 p$ & - & 1 & 0 & 4 \\
\hline 1268-08 [NP] & Cu 2p & - & 1 & 0 & 4 \\
\hline 1268-09 [NP] & Cu $2 p$ & - & 1 & 0 & 4 \\
\hline
\end{tabular}

*Voltage shift of the archived (as-measured) spectrum relative to the printed figure. The figure reflects the recommended energy scale correction due to a calibration correction, sample charging, flood gun, or other phenomenon.

** [NP] signifies not published; digital spectra are archived in SSS database but not reproduced in the printed journal.

1. $\mathrm{Be}$

2. $\mathrm{BeO}$

3. Au calibration

4. Cu calibration 


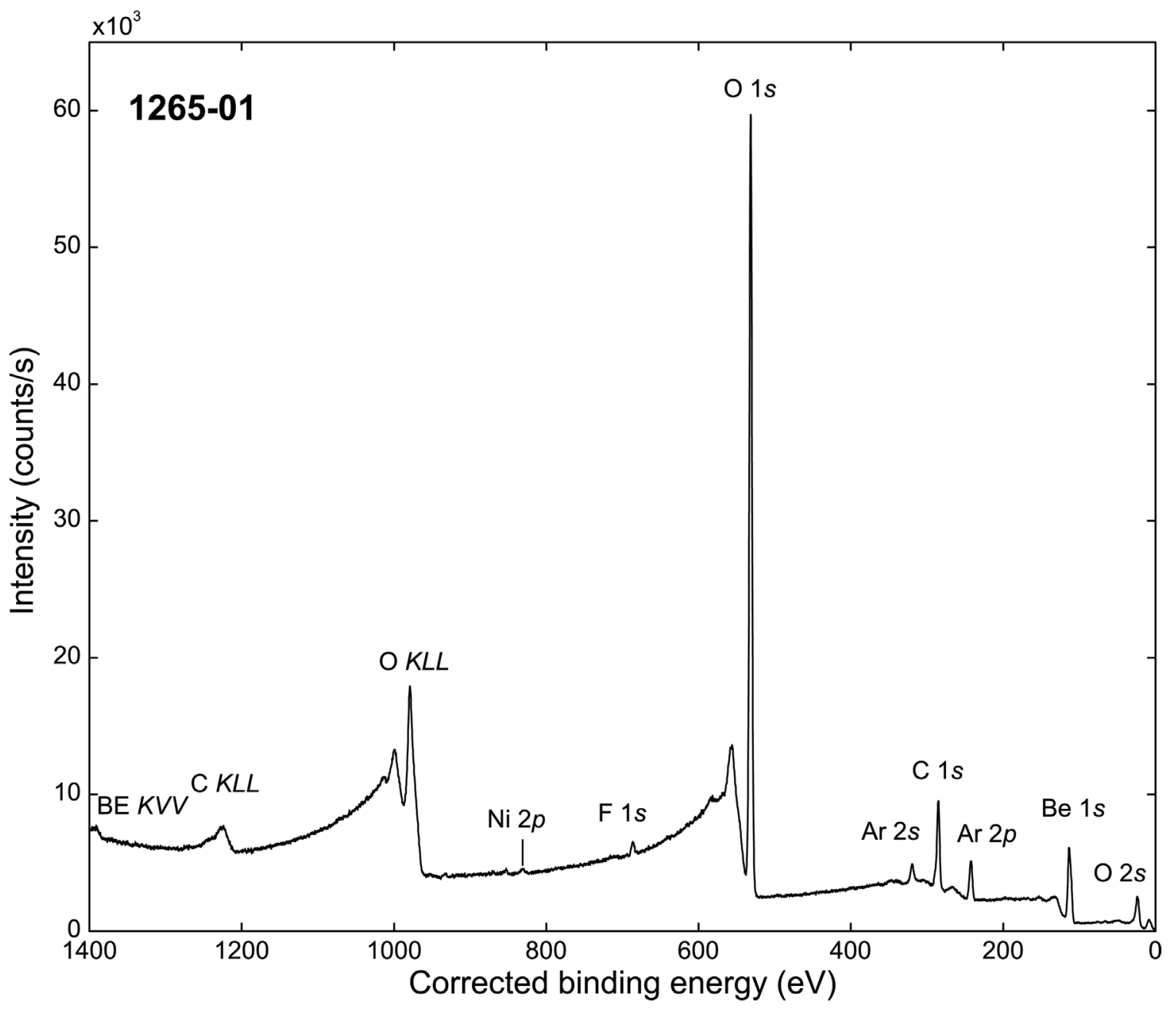

\begin{tabular}{rc}
\hline Accession \# & 01265-01 \\
Host Material & Be foil \\
Technique & XPS \\
Spectral Region & survey \\
Instrument & Excitation Source \\
Source Energy & Thermo Scientific Theta Probe \\
Source Strength & Al $K_{\alpha}$ monochromatic \\
Source Size & $1486.6 \mathrm{eV}$ \\
Analyzer Type & $200 \mathrm{~W}$ \\
Incident Angle & $0.2 \mathrm{~mm} \times 0.2 \mathrm{~mm}$ \\
Emission Angle & spherical sector \\
Analyzer Pass Energy: & $30^{\circ}$ \\
Analyzer Resolution & $53^{\circ}$ \\
Total Elapsed Time & $300 \mathrm{eV}$ \\
Number of Scans & $2.10 \mathrm{eV}$ \\
Total Signal Accumulation Time & $2333 \mathrm{~s}$ \\
Source Beam Size at Specimen Surface & $6960 \mathrm{~s}$ \\
Effective Detector Width & 10 \\
\hline
\end{tabular}




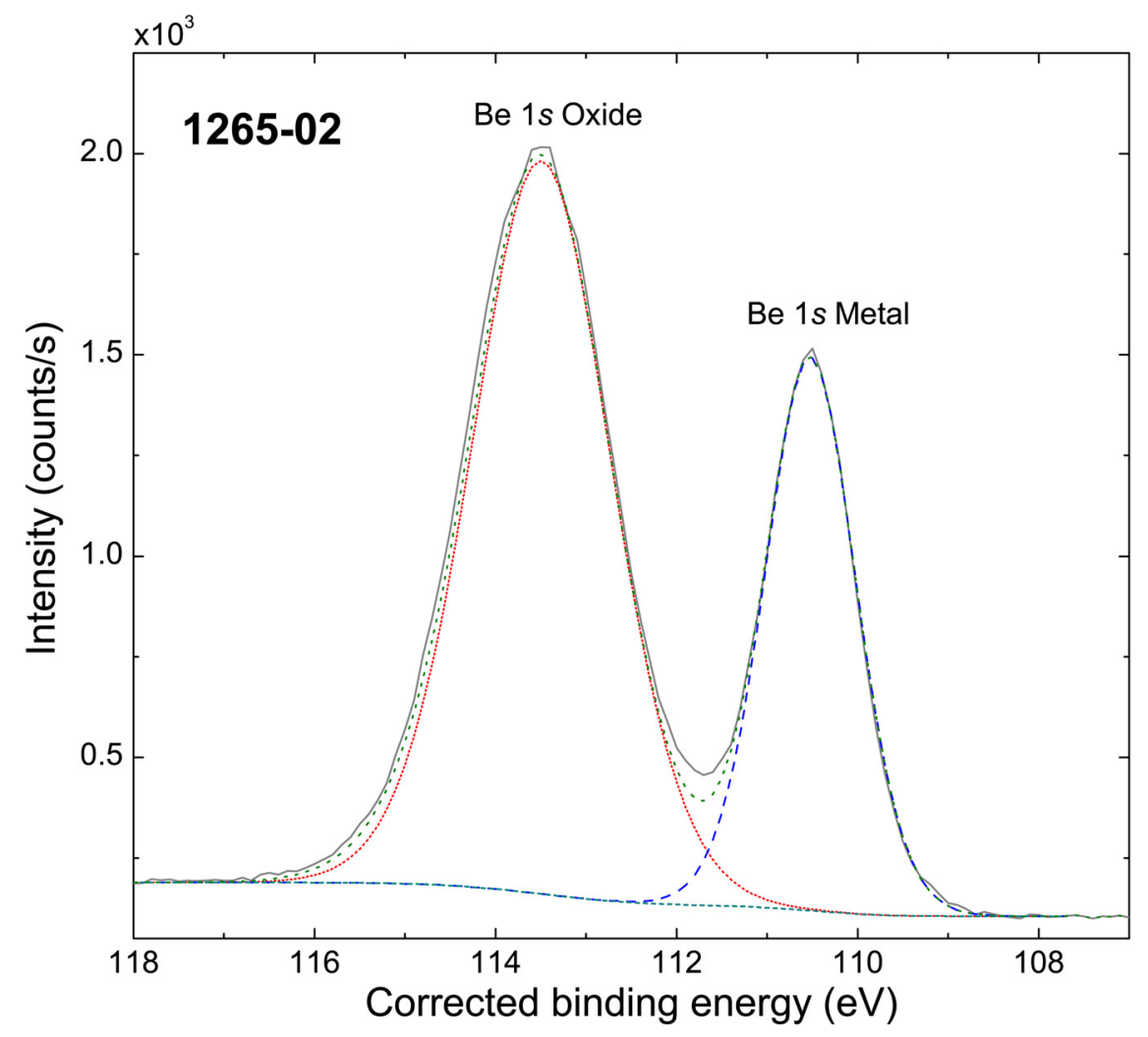

Accession \#: 01265-02

- Host Material: Be foil

- Technique: XPS

- Spectral Region: Be $1 s$

Instrument: Thermo Scientific Theta Probe

Excitation Source: Al $K_{\alpha}$ monochromatic

Source Energy: $1486.6 \mathrm{eV}$

Source Strength: $200 \mathrm{~W}$

Source Size: $0.2 \mathrm{~mm} \times 0.2 \mathrm{~mm}$

Analyzer Type: spherical sector Incident Angle: $30^{\circ}$

Emission Angle: $53^{\circ}$

Analyzer Pass Energy: $80 \mathrm{eV}$

Analyzer Resolution: $0.80 \mathrm{eV}$

Total Signal Accumulation Time: $2257 \mathrm{~s}$

Total Elapsed Time: $2708 \mathrm{~s}$

Number of Scans: 150

Source Beam Size at Specimen Surface: $0.4 \mathrm{~mm} \times 0.4 \mathrm{~mm}$ Effective Detector Width: $0.1 \mathrm{eV}$

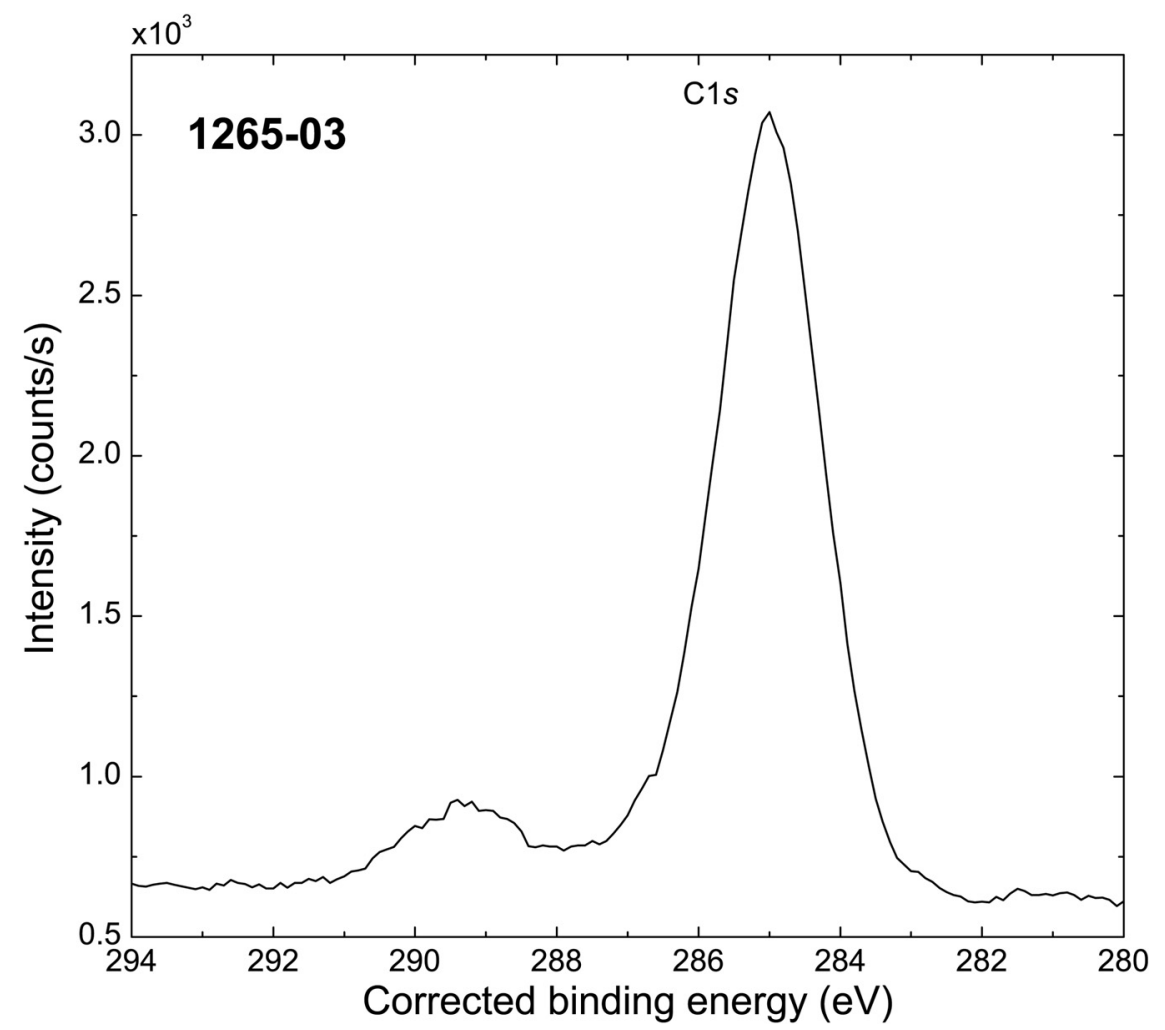

- Accession \#: 01265-03

- Host Material: Be foil

- Technique: XPS

- Spectral Region: C $1 \mathrm{~s}$

Instrument: Thermo Scientific Theta Probe

Excitation Source: Al $K_{\alpha}$ monochromatic

Source Energy: $1486.6 \mathrm{eV}$

Source Strength: $200 \mathrm{~W}$

Source Size: $0.2 \mathrm{~mm} \times 0.2 \mathrm{~mm}$

Analyzer Type: spherical sector Incident Angle: $30^{\circ}$

Emission Angle: $53^{\circ}$

Analyzer Pass Energy: $80 \mathrm{eV}$

Analyzer Resolution: $0.80 \mathrm{eV}$

Total Signal Accumulation Time: $1005 \mathrm{~s}$

Total Elapsed Time: $1460 \mathrm{~s}$

Number of Scans: 100

Source Beam Size at Specimen Surface: $0.4 \mathrm{~mm} \times 0.4 \mathrm{~mm}$

Effective Detector Width: $0.1 \mathrm{eV}$ 


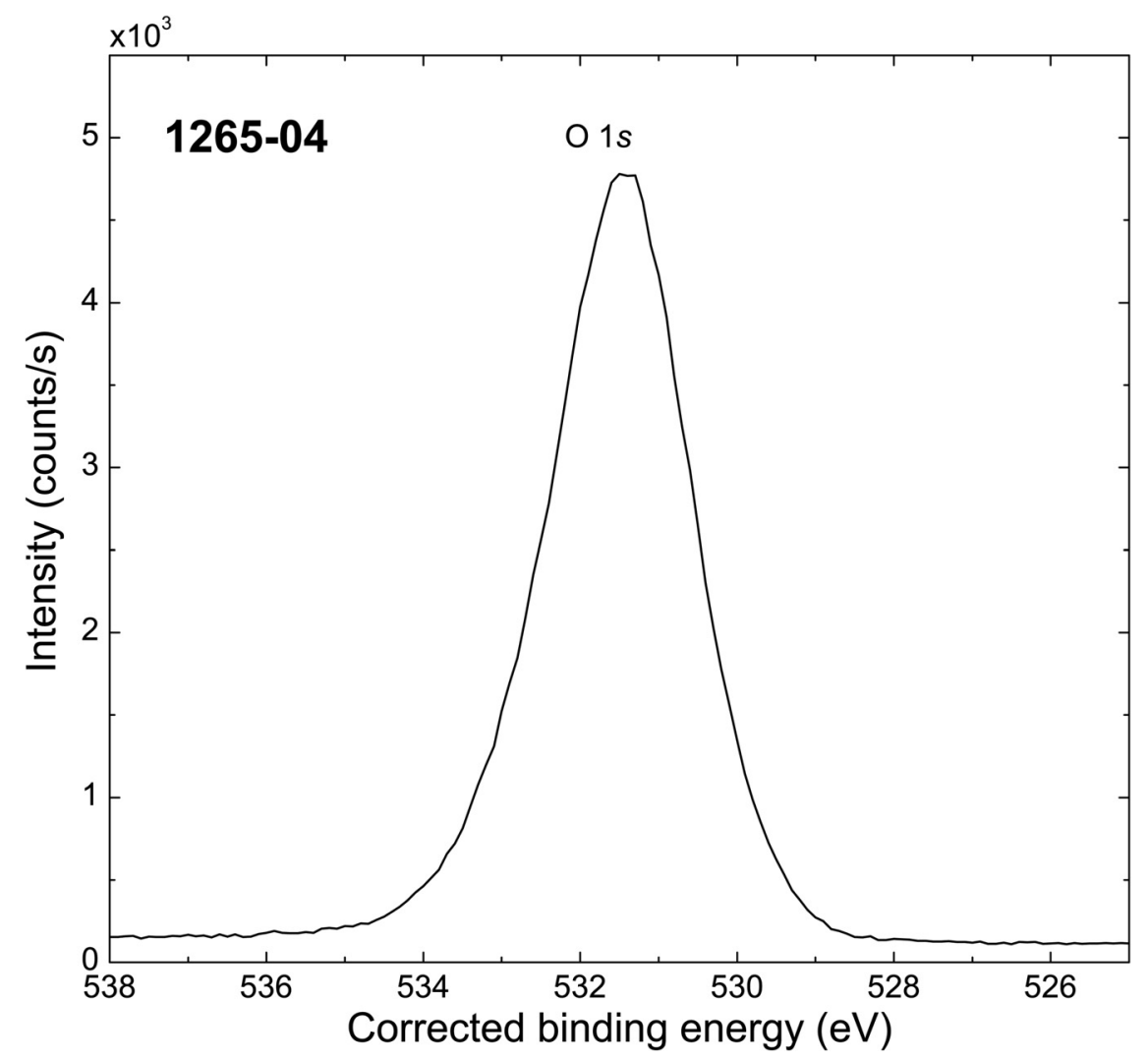

- Accession \#: 01265-04

- Host Material: Be foil

- Technique: XPS

- Spectral Region: $01 \mathrm{~s}$

Instrument: Thermo Scientific Theta Probe

Excitation Source: Al $K_{\alpha}$ monochromatic

Source Energy: $1486.6 \mathrm{eV}$

Source Strength: $200 \mathrm{~W}$

Source Size: $0.2 \mathrm{~mm} \times 0.2 \mathrm{~mm}$

Analyzer Type: spherical sector

Incident Angle: $30^{\circ}$

Emission Angle: $53^{\circ}$

Analyzer Pass Energy: $30 \mathrm{eV}$

Analyzer Resolution: $0.60 \mathrm{eV}$

Total Signal Accumulation Time: $1005 \mathrm{~s}$

Total Elapsed Time: $1483 \mathrm{~s}$

Number of Scans: 100

Source Beam Size at Specimen Surface: $0.4 \mathrm{~mm} \times 0.2 \mathrm{~mm}$

Effective Detector Width: $0.1 \mathrm{eV}$

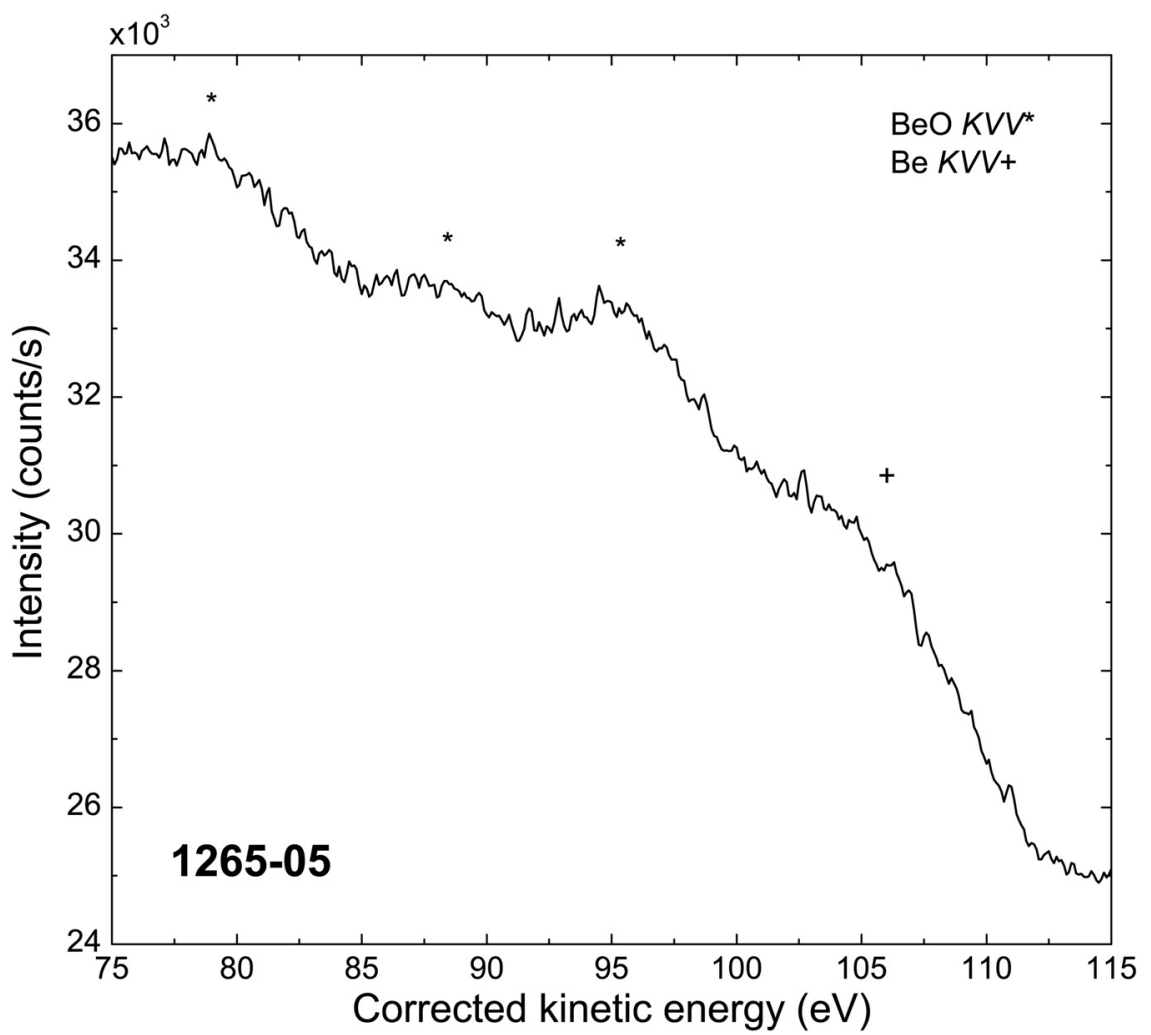

- Accession \#: 01265-05

- Host Material: Be foil

- Technique: XPS

- Spectral Region: Be KVV

Instrument: Thermo Scientific Theta Probe

Excitation Source: Al $K_{\alpha}$ monochromatic

Source Energy: $1486.6 \mathrm{eV}$

Source Strength: $200 \mathrm{~W}$

Source Size: $0.2 \mathrm{~mm} \times 0.2 \mathrm{~mm}$ Analyzer Type: spherical sector Incident Angle: $30^{\circ}$

Emission Angle: $53^{\circ}$

Analyzer Pass Energy: $300 \mathrm{eV}$ Analyzer Resolution: $2.10 \mathrm{eV}$

Total Signal Accumulation Time: $1691 \mathrm{~s}$

Total Elapsed Time: $4518 \mathrm{~s}$

Number of Scans: 75

Source Beam Size at Specimen Surface: $0.4 \mathrm{~mm} \times 0.2 \mathrm{~mm}$

Effective Detector Width: $0.1 \mathrm{eV}$ 


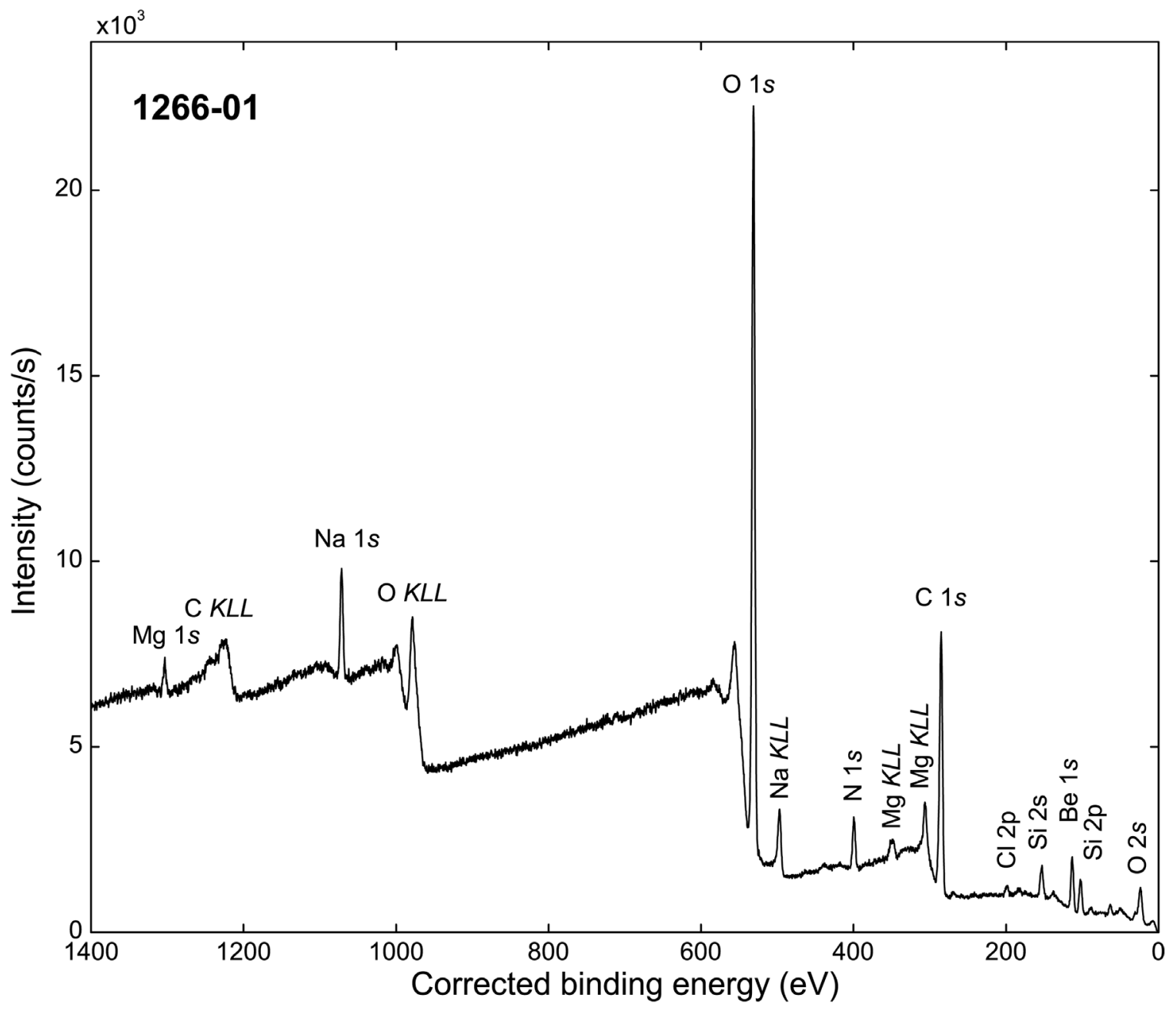

\begin{tabular}{rc}
\hline Accession \# & 01266-01 \\
Host Material & BeO foil \\
Technique & XPS \\
Spectral Region & survey \\
Instrument & Thermo Scientific Theta Probe \\
Source Energy & Al $K_{\alpha}$ monochromatic \\
Source Strength & $1486.6 \mathrm{eV}$ \\
Source Size & $200 \mathrm{~W}$ \\
Analyzer Type & $0.2 \mathrm{~mm} \times 0.2 \mathrm{~mm}$ \\
Incident Angle & spherical sector \\
Emission Angle & $30^{\circ}$ \\
Analyzer Pass Energy: & $53^{\circ}$ \\
Analyzer Resolution & $300 \mathrm{eV}$ \\
Total Elapsed Time & $2.10 \mathrm{eV}$ \\
Number of Scans & $2333 \mathrm{~s}$ \\
Total Signal Accumulation Time & $6960 \mathrm{~s}$ \\
Effective Detector Width & 10 \\
Source Beam Size at Specimen Surface & $0.4 \mathrm{~mm} \times 0.2 \mathrm{~mm}$ \\
\hline
\end{tabular}




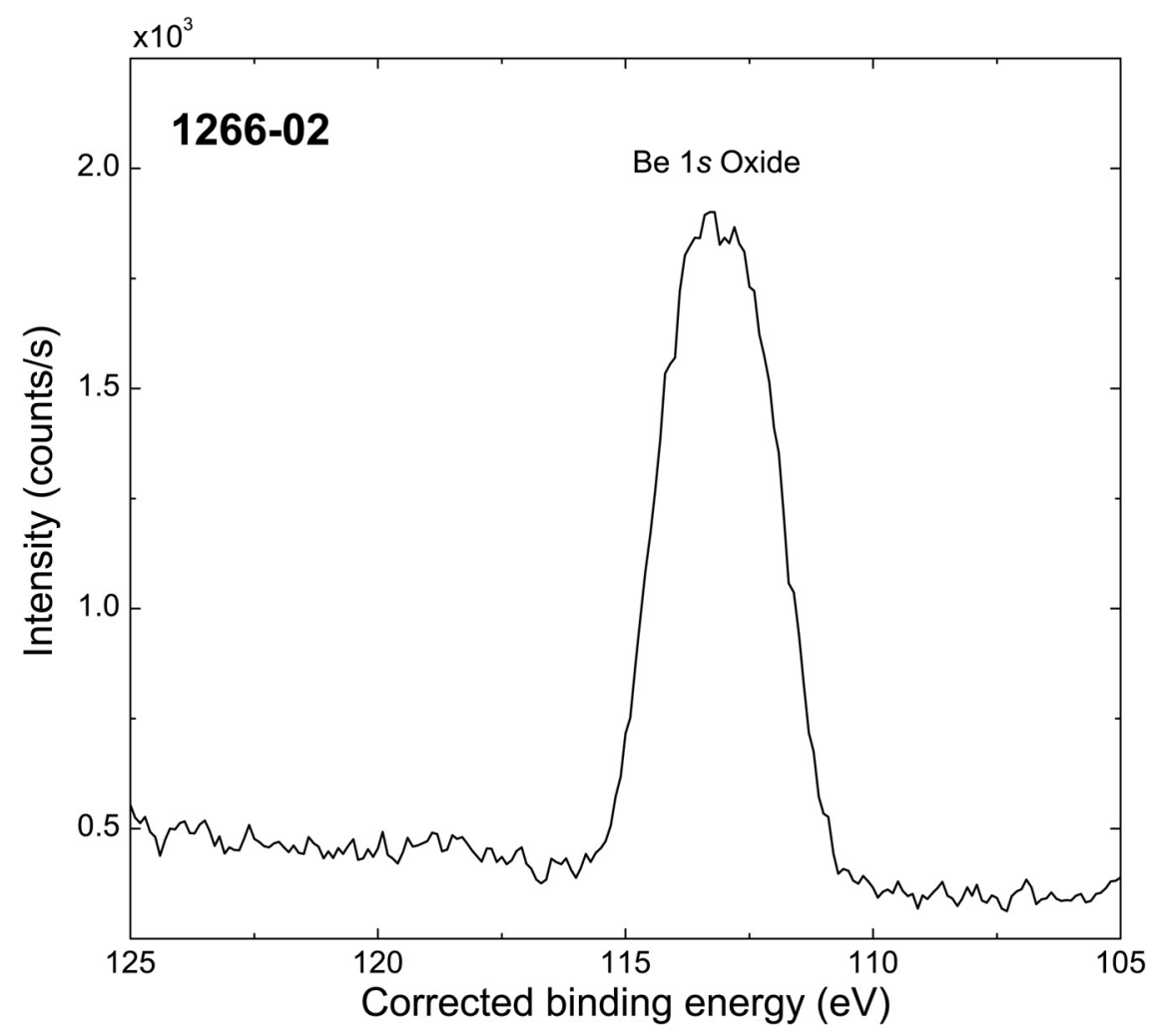

- Accession \#: 01266-02

- Host Material: $\mathrm{BeO}$ foil

- Technique: XPS

- Spectral Region: Be $1 s$

Instrument: Thermo Scientific Theta Probe

Excitation Source: Al $K_{\alpha}$ monochromatic

Source Energy: $1486.6 \mathrm{eV}$

Source Strength: $200 \mathrm{~W}$

Source Size: $0.2 \mathrm{~mm} \times 0.2 \mathrm{~mm}$ Analyzer Type: spherical sector Incident Angle: $30^{\circ}$

Emission Angle: $53^{\circ}$

Analyzer Pass Energy: $80 \mathrm{eV}$ Analyzer Resolution: $0.80 \mathrm{eV}$ Total Signal Accumulation Time: $526 \mathrm{~s}$

Total Elapsed Time: $840 \mathrm{~s}$

Number of Scans: 40

Source Beam Size at Specimen Surface: $0.4 \mathrm{~mm} \times 0.4 \mathrm{~mm}$

Effective Detector Width: $0.1 \mathrm{eV}$

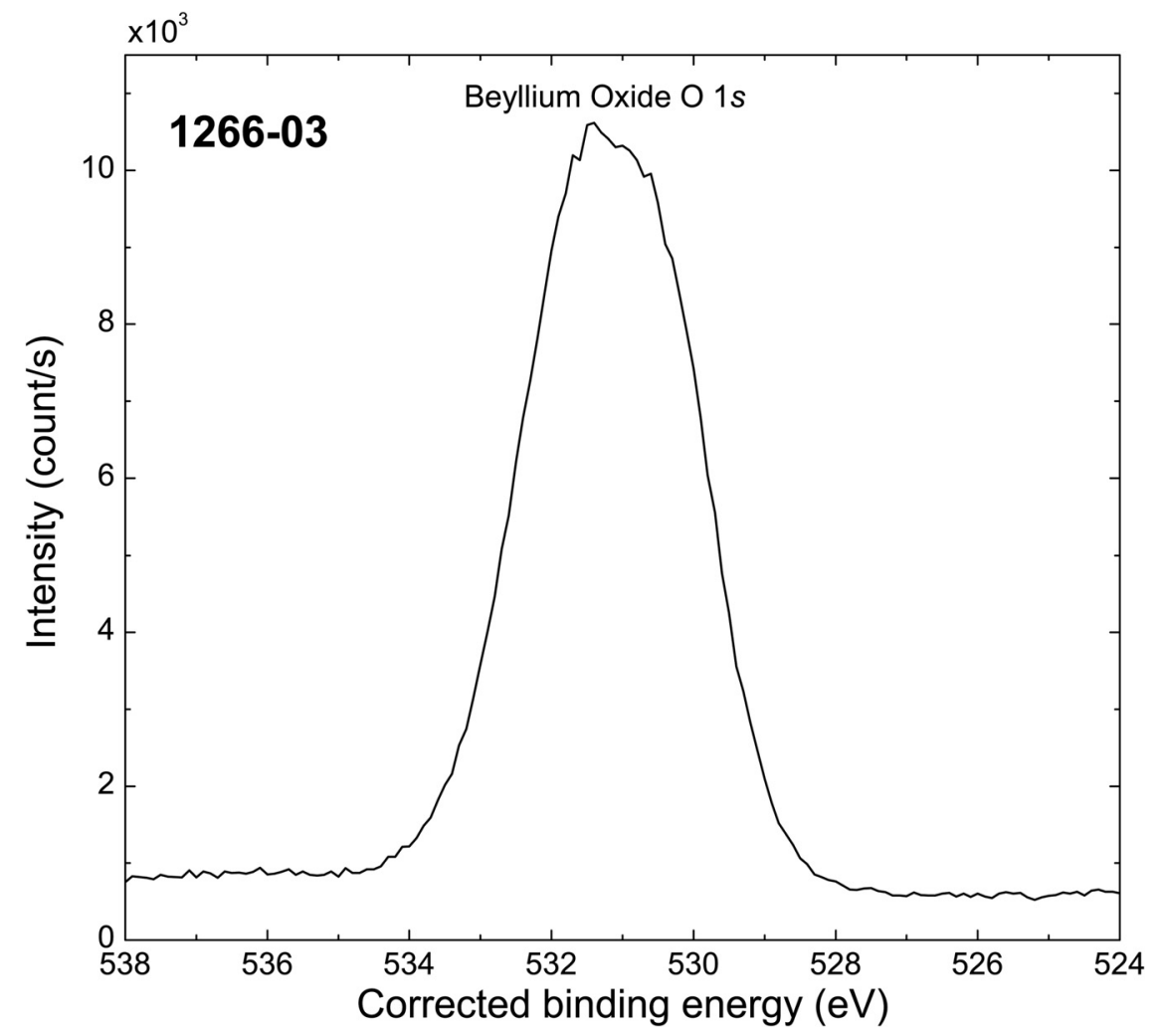

Accession \#: 01266-03

- Host Material: $\mathrm{BeO}$ foil

- Technique: XPS

- Spectral Region: $01 \mathrm{~s}$

Instrument: Thermo Scientific Theta Probe

Excitation Source: Al $K_{\alpha}$ monochromatic

Source Energy: $1486.6 \mathrm{eV}$

Source Strength: $200 \mathrm{~W}$

Source Size: $0.2 \mathrm{~mm} \times 0.2 \mathrm{~mm}$

Analyzer Type: spherical sector Incident Angle: $30^{\circ}$

Emission Angle: $53^{\circ}$

Analyzer Pass Energy: $50 \mathrm{eV}$ Analyzer Resolution: $0.65 \mathrm{eV}$ Total Signal Accumulation Time: $526 \mathrm{~s}$

Total Elapsed Time: $860 \mathrm{~s}$

Number of Scans: 30

Source Beam Size at Specimen Surface: $0.4 \mathrm{~mm} \times 0.2 \mathrm{~mm}$

Effective Detector Width: $0.1 \mathrm{eV}$ 


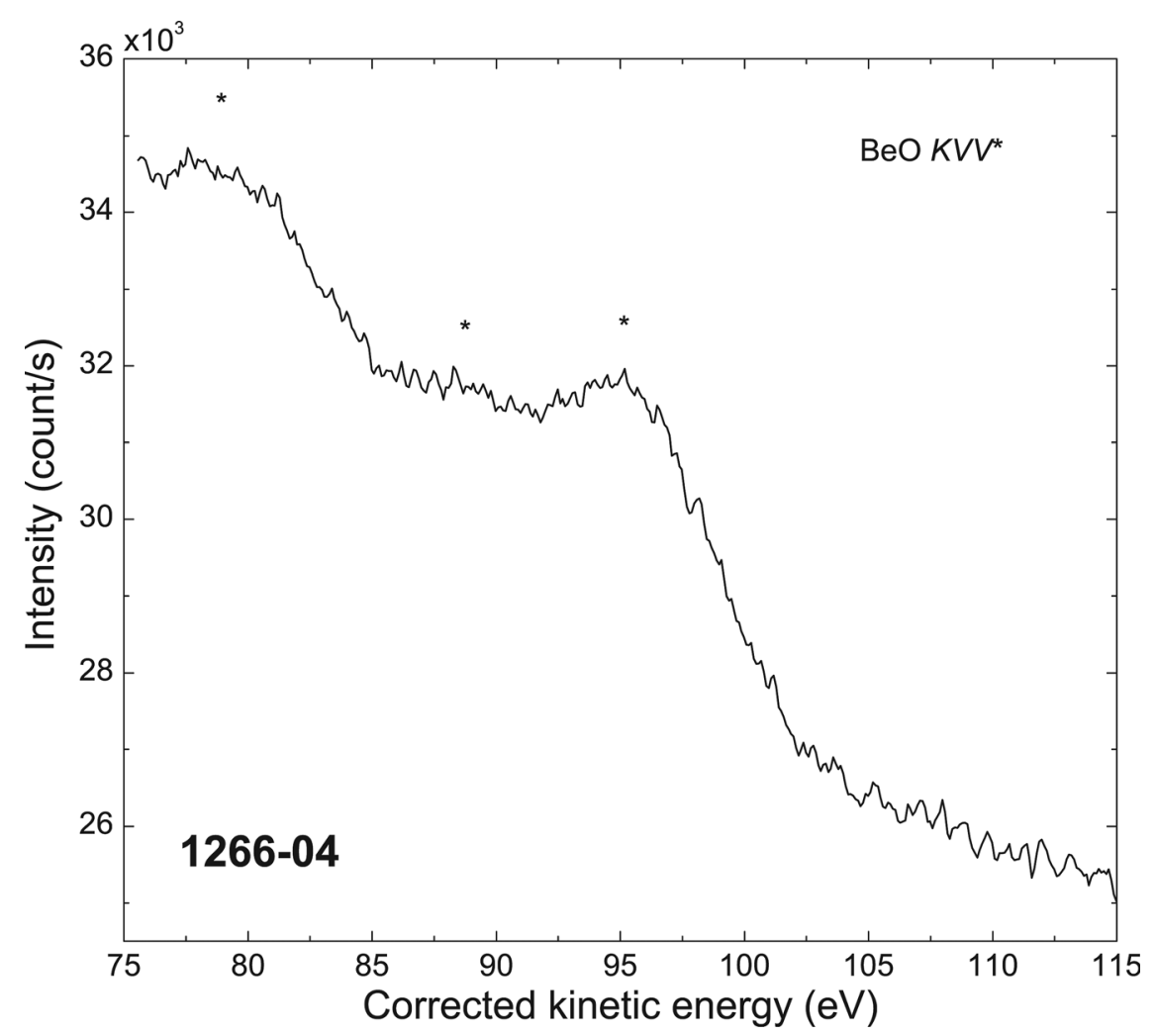

- Accession \#: 01266-04

- Host Material: $\mathrm{BeO}$ foil

- Technique: XPS

- Spectral Region: Be KVV

Instrument: Thermo Scientific Theta Probe

Excitation Source: Al $K_{\alpha}$ monochromatic

Source Energy: $1486.6 \mathrm{eV}$

Source Strength: $200 \mathrm{~W}$

Source Size: $0.2 \mathrm{~mm} \times 0.2 \mathrm{~mm}$ Analyzer Type: spherical sector Incident Angle: $30^{\circ}$

Emission Angle: $53^{\circ}$

Analyzer Pass Energy: $300 \mathrm{eV}$

Analyzer Resolution: $2.10 \mathrm{eV}$

Total Signal Accumulation Time: $802 \mathrm{~s}$

Total Elapsed Time: $1249 \mathrm{~s}$

Number of Scans: 40

Source Beam Size at Specimen Surface: $0.4 \mathrm{~mm} \times 0.2 \mathrm{~mm}$

Effective Detector Width: $0.1 \mathrm{eV}$ 